\title{
Publisher's Note: Robust determination of the superconducting gap sign structure via quasiparticle interference [Phys. Rev. B 92, 184513 (2015)]
}

\author{
P. J. Hirschfeld, D. Altenfeld, I. Eremin, and I. I. Mazin \\ (Received 4 November 2016; published 11 November 2016)
}

DOI: 10.1103/PhysRevB.94.179905

This paper was published online on 30 November 2015 with an incorrect Grant number in the Acknowledgments. The second sentence in the Acknowledgments should read as "P.J.H. was supported by NSF-DMR-1407502, and I.I.M. by the U.S. Office of Naval Research through the Naval Research Laboratory's Basic Research Program." The Acknowledgments have been corrected as of 4 November 2016. The Acknowledgments are incorrect in the printed version of the journal. 